\title{
Dry Eye Disease: Early Recognition with Guidance on Management and Treatment for Primary Care Family Physicians
}

\author{
Mohamud A. Verjee (D) · Ashley R. Brissette · Christopher E. Starr (D
}

Received: July 17, 2020 / Accepted: September 24, 2020 / Published online: October 22, 2020

(C) The Author(s) 2020

\begin{abstract}
Primary care presentations of dry eye disease (DED) are common and pose a diagnostic challenge due to the variety of symptoms and the absence of certainty for family practitioners. While there are many published articles on the topic, the 2017 Tear Film and Ocular Surface Society Dry Eye Workshop was a landmark report in distinguishing multifactorial differences. Redefined terms clarified the DED disorder. The ocular surface-the tear/air interfaceis the primary refractive component of the eye, which is why DED is so significant and impacts vision. There is a high prevalence of DED in the community, ranging from $5 \%$ to $30 \%$ of people across multiple studies. Elderly patients have up to $75 \%$ increased risk of DED and receive more intensive treatment than younger age groups. DED is also more common in women than men,
\end{abstract}

Electronic supplementary material The online version of this article (https://doi.org/10.1007/s40123020-00308-z) contains supplementary material, which is available to authorized users.

M. A. Verjee $(\square)$

Medical Student Affairs, \& Medical Education, Weill

Cornell Medicine - Qatar, Doha, Qatar

e-mail: mov2002@qatar-med.cornell.edu

A. R. Brissette - C. E. Starr

Department of Ophthalmology, Weill Cornell

Medicine, New York, NY, USA occurring in $9.8 \%$ of postmenopausal women. The causes of DED span defective lacrimal apparatus and systemic disorders. Despite its prevalence, up to one-half of patients with confirmed DED do not receive proper alleviating treatment. Risk factors on functional and environmental bases follow. Tools to elicit a diagnosis more confidently are outlined using the Ocular Surface Disease Index (OSDI) and the Symptom Assessment in Dry Eye questionnaires (SANDE). Lacritin, lutein, vitamin A, and balanced nutrition are essential contributors to maintaining healthy eyes with appropriate management and treatment. The authors hope that this paper will prompt a more accurate and expedient diagnosis of DED in primary care practice and an earlier recognition of specialist referrals.

\section{PLAIN LANGUAGE SUMMARY}

Dry eye disease (DED) poses a diagnostic challenge to primary care physicians. The condition involves the tear/air interface, at the corneal (ocular) surface, where light enters the eye. Any change in light refraction affects regular sight. Symptoms of DED include itching, grittiness, foreign body sensation, redness, excessive tearing, and visual blurring, the lattermost being the most common presentation. Ultimately, the 
untreated disease leads to continued discomfort and visual deficit, but when severe, it can result in blindness. Up to $30 \%$ of the population suffers from DED, with the elderly, particularly women, more affected. Unfortunately, up to $50 \%$ of those affected have inadequate treatment. Questionnaires help in diagnosis. Simple office tests can indicate the severity of eye problems. Technology with smartphone photography can take high-resolution images, which are useful in education and teaching. A multitude of conditions cause DED, including blepharitis, an infection of the eyelids. Environmental risks also abound. Proper nutrition is essential in maintaining eye health. Intermittent eye symptoms are likely to be underrated by the public, by purchasing over-the-counter products such as artificial tears rather than seeking a proper medical check. Newer prescription medications are now available for DED relief before advancing to debility requiring specialist treatment.

Keywords: Biofilm; Blepharitis; Dry eye disease (DED); Lacritin; Lutein; Meibomian gland dysfunction (MGD); Ocular surface; Tear film

\section{Key Summary Points}

Why carry out this study?

A notable lack of awareness of DED at the first point of physician contact reported by other authors, without prompt diagnosis of the condition, results in greater patient discomfort and ultimately visual damage.

Approximately half of all patients diagnosed with the condition do not receive the correct treatment in primary care (FP) settings.

\section{What was learned from the study?}

This paper articulates and summarizes the TFOS DEWS II findings succinctly to enable a better understanding of the diagnosis, management, and treatment of DED by primary care (FP) family physicians.
Office procedures are simple and practical for either confirmation or exclusion of DED, together with a deeper understanding of presenting symptoms and recognizing a significant cause of visual morbidity.

\section{DIGITAL FEATURES}

This article is published with digital features, including a summary slide, plain language summary, and video, to facilitate understanding of the article. To view digital features for this article go to https://doi.org/10.6084/m9. figshare.12994019.

\section{BACKGROUND: OCULAR SURFACE}

A tear film overlies all ocular surfaces. This film was previously thought to comprise three distinct layers: an outer oily lipid layer, a middle dominant aqueous layer, and an inner mucinous layer. However, the tear film likely exists as a bilayer with an outer lipid layer and an inner mixed aqueous/mucinous fluid layer. These layers maintain hydration status and protect the ocular surface. Disruption of any of the layers can damage this surface and cause pain to become a prominent symptom.

\section{UNDERSTANDING DRY EYE DISEASE}

Dry eye disease (DED) and dry eye syndrome (DES) are sometimes used synonymously. Craig et al. [1] redefined an evidence-based and contemporary DED classification drawn up from the Tear Film \& Ocular Surface Society Dry Eye Workshop II (TFOS DEWS) meeting in 2007 [2], recognizing its multifactorial nature. TFOS DEWS II [1] revised the definition of DED as:

"a multifactorial disease of the ocular surface characterized by a loss of homeostasis 
of the tear film, and accompanied by ocular symptoms, in which tear film instability and hyperosmolarity, ocular surface inflammation and damage, and neurosensory abnormalities play etiological roles."

The report guided clinical management and research [1]. The new DED description updated the disorder, presenting a three-part classification system: dry eye problems are disturbances of the lacrimal functional unit (LFU) comprising the

1. Lacrimal glands

2. Ocular surface

3. Eyelids

While the terms "aqueous deficient" and "evaporative dry eye" were removed from the new definition, they remain valid within the etiological classification [1].

DED causes a chronic lack of sufficient lubrication to the eye's aqueous tear film layer, leading to the ocular surface's disruption. DED is a multifactorial disorder with inflammation, tear evaporation, and increased osmolarity as its core pathophysiologic processes [1, 2]. Keratoconjunctivitis sicca (KCS) does have a dry eye component but should not be mistaken for DED. Its use is now limited to Sjögren's syndrome. The Delphi panel in 2006 emphasized that tear dysfunction involves more than aqueous deficiency. The majority of DED may be attributed to the tear film's insufficient lipid layer, leading to excessive tear evaporation [3]. Lack of lacrimal gland activity leads to aqueous tear deficiency (ATD) [1], but an evaporative loss, both intrinsic and extrinsic, also has to be considered. Elevated levels of tear nerve growth factor (NGF) found in those suffering from KCS may have a role in surface inflammation. Prolonged DED may lead to squamous metaplasia, reduction of mucin-producing goblet cells, as well as hyperkeratosis. Hormonal changes during menopause and pregnancy, and cataract and refractive surgery may also adversely impact lacrimal activity.

Assessing eyelid health when DED is confirmed is important as there is a close association with blepharitis and Meibomian gland dysfunction (MGD). Rynerson and Perry [4] coined the new term of dry eye blepharitis syndrome (DEBS). A biofilm structure is a target for colonizing bacteria. The invasion initiates quorum-sensing gene activation, subsequently producing inflammatory "virulence factors", including super-antigens, which persist for a lifetime. These factors cause follicular inflammation, MGD, aqueous deficiency (lacrimalitis), with lid damage and destruction sustained in the long term. Lower eyelid surface appearances are visible, initially with scurf, also known as cylindrical dandruff, later undergoing keratinization, as seen in Figs. 1 and 2 [5, 6]. Meibography illustrates MGD changes as seen in Fig. 3 [7]. In more advanced stages, eyelid destruction is often accompanied by an ectropion or entropion or may cause an eyelid to become floppy.

\section{COMPLIANCE WITH ETHICS GUIDELINES}

This article is based on previously conducted studies and does not contain any studies with human participants or animals performed by any of the authors.

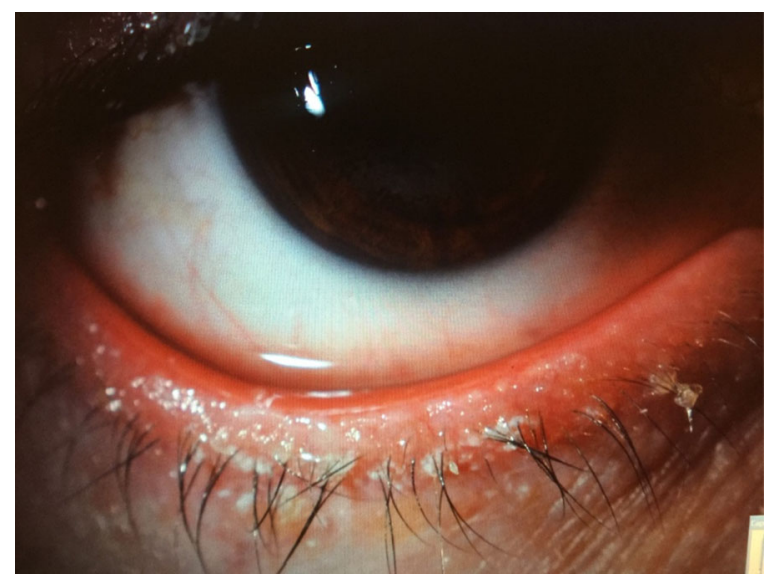

Fig. 1 Anterior blepharitis showing scurf. Copyright (C) 2020 Starr C E. Weill Cornell Medicine, New York, NY, USA 


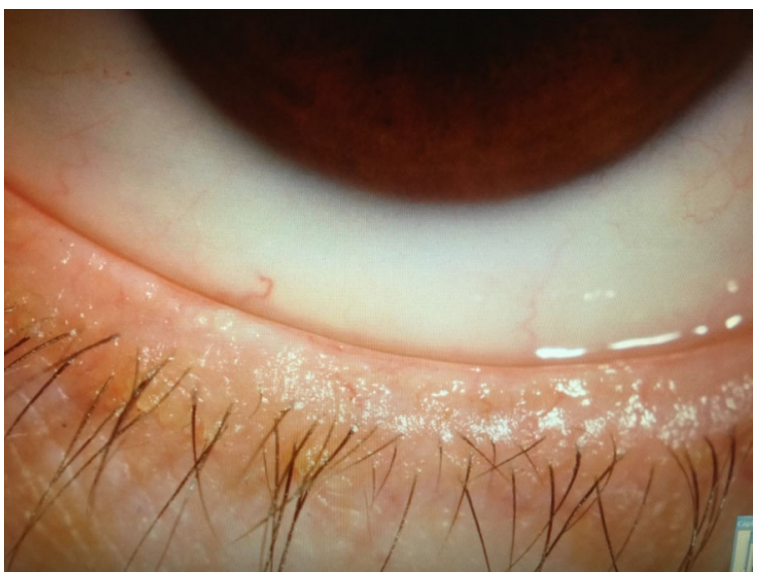

Fig. 2 Anterior blepharitis showing keratinization. Copyright (C) 2020 Starr C E. Weill Cornell Medicine, New York, NY, USA

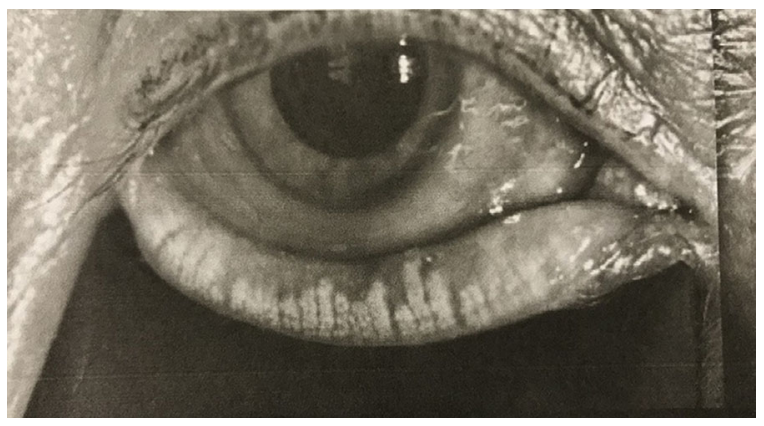

Fig. 3 Meibography showing Meibomian gland dysfunction (MGD); note darker areas on the lower lid signifying the loss. Copyright (C) 2020 Starr C E. Weill Cornell Medicine, New York, NY, USA

\section{PREVALENCE}

The prevalence of DED is estimated to be $5-50 \%$, depending upon comorbidities. The incidence in postmenopausal women increases from $5.7 \%$ at younger than 50 years to $9.8 \%$ and up to $75 \%$ from age 75 years $[8,9]$. Many people are unaware of their underlying condition. Of the estimated 50 million people with DED in the USA, approximately 35 million are undiagnosed [3]. Aging is a natural process in which the symptoms and signs of DED may be disregarded or overlooked by a patient, without relating the problem to their physician of care.

\section{CAUSES OF DED}

\section{Ocular Surface Dysfunction}

Gupta et al. [10] found that ocular surface dysfunction (OSD) was common in patients presenting for cataract surgery, although many presented undiagnosed. Because of its high prevalence, screening was suggested with tear testing before invasive surgery.

Starr et al. [11] wrote a seminal paper on DED and ocular surgery, noting the impact on OSD after any surgical procedure: DED symptoms could be exacerbated, resulting in decreased vision and postoperative dissatisfaction. The American Society of Cataract and Refractive Surgery (ASCRS) addressed the lack of awareness on surgical outcomes by developing a new consensus-based OSD algorithm to elicit and treat visually significant OSD before any refractive surgery commenced. Their objective was to improve postoperative visual outcomes and enhance patient satisfaction.

\section{Blink Rate}

Patients with Parkinson's disease are vulnerable to DED because of a low blink reflex and poor tear film distribution over the ocular surface. People with Parkinson's disease may have a severely decreased blink rate of only two blinks per minute versus an average "normal" blink rate of up to 18 per minute [12]. Wolffsohn et al. [13] evaluated a patient self-administered Dry Eye Blink Test against established DED diagnosis criteria and found it offered a rapid method of identifying possible DED. The test involved a patient observing an image on a computer screen while trying not to blink after administering a simple over-the-counter eye isotonic solution. The length of time, measured in seconds, of blink absence without discomfort was measured with a 10-s cutoff. This screening method, combined with a questionnaire, showed a sensitivity of $71 \%$, and a $90 \%$ specificity for diagnosing DED. 


\section{Autoimmune Diseases and Other Disorders}

Rheumatic diseases can present with KCS. Signs of anterior uveitis in rheumatoid arthritis and symptoms of systemic lupus erythematosus (SLE), scleroderma, and relapsing granulomatosis, with polyangiitis (GPA), formerly known as Wegener's granulomatosis are important. Other autoimmune diseases to eliminate are Sjögren's syndrome (SjS), Behçet's syndrome, hyperthyroidism with irreversible proptosis in Graves' disease, rarely dermatomyositis, connective tissue diseases, trachoma, eyelid disorders, and vitamin A deficiency. MGD, ectropion, tricyclic antidepressants, antihistamines, mydriatics, oral contraceptives, diuretics, and antihypertensive medications, including beta-blockers, predispose to an increased likelihood of DED. Allergic conjunctivitis also affects the ocular surface (OS), altering the tear film layer. Narrowing or, rarely, the congenital absence of a lacrimal duct opening causes a lacrimal deficiency [1]. Recall that anterior blepharitis and MGD, involving inflammation of the eyelid margins, are closely associated with DED. Untreated, prolonged blepharitis can lead to severe destruction of eyelid integrity.

\section{SYMPTOMS TO NOTE}

DED symptoms include eye irritation leading to itching, grittiness, foreign body sensation, redness, excessive periodic tearing or reflex epiphora, and temporary visual blurring, and many are also common in blepharitis. Careful examination of the lid margins can help to differentiate between the two entities. Intermittent or fluctuating blurry vision is the most common presenting symptom of DED. Sharp stabbing, burning, or stinging pains with sudden tearing cause distress alongside a patient's rationalization of a foreign body, such as a loose eyelash or debris being responsible. Eyes are sensitive owing to the high concentration of ocular surface nerve endings. The cause of this extreme pain is minor shearing of dried OS cells

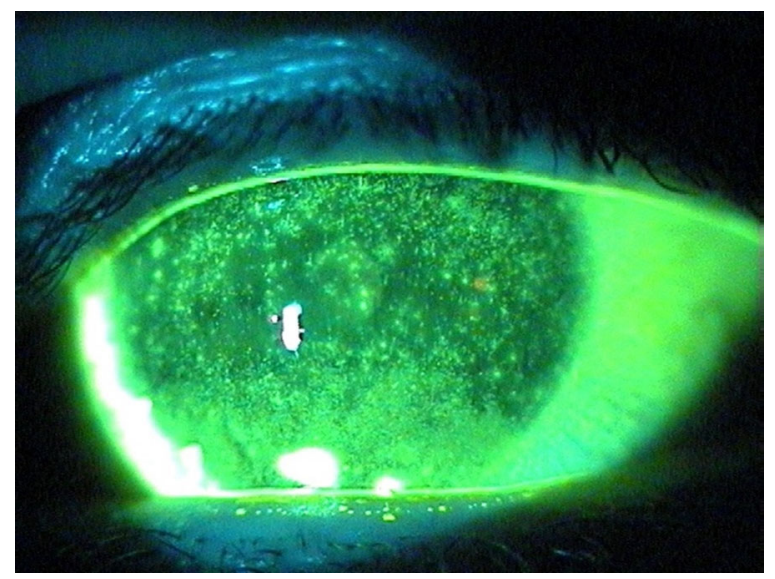

Fig. 4 Dry eye disease: fluorescein stain highlighting widespread ocular surface damage. Permission for reproduction granted. Copyright (C) 2020 American Academy of Ophthalmology

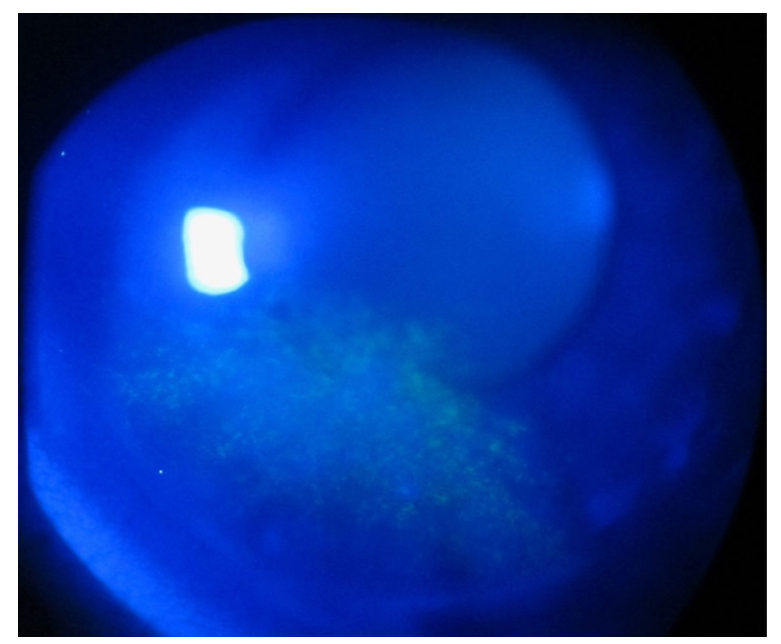

Fig. 5 Dry eye disease: fluorescein staining showing localized ocular surface damage. Copyright (C) 2020 Starr C E. Weill Cornell Medicine, New York

and hyperstimulation of corneal sensory nerves, with surface damage seen in Figs. 4 and 5 $[14,15]$. Distracting pain may continue for hours before abating with a visible red eye. Anterior blepharitis and MGD, involving inflammation of the eyelid margins, are often associated with evaporative DED. These significant symptoms of DED can lead to reduced quality of life and lost work productivity. 
A quality of life matter to consider arose when $\mathrm{Na}$ et al. [16] found a close association between DED, stress, and depression in those clinically diagnosed and presenting with eye symptoms. Schiffman et al. [17] used utility measurements (patient preferences) and found an equivalent value for DED and moderate-tosevere angina. Patients completed the interactive software utility assessment questionnaires by the time trade-off (TTO) method. Utility scores were scaled such that a score of 1.0 signified perfect health and 0 was equivalent to death. Utility scores for DED ranged from 0.78 to 0.72 , and 0.75 to 0.71 for angina. DED symptomatology can be seen to have a meaningful impact on the disruption of health.

\section{DRY EYE QUESTIONNAIRE (DEQ-5), OCULAR SURFACE DISEASE INDEX (OSDI), AND OTHER QUESTIONNAIRES FOR CONFIRMING DED}

Two validated questionnaires-DEQ-5 and the OSDI-were recommended in TFOS DEWS II $[1,18]$. Symptom Assessment in Dry Eye (SANDE), Standard Patient Evaluation of Eye Dryness (SPEED) questionnaire, and others are used in research and general optometry and ophthalmology practices. Chalmers et al. [19] validated the DEQ-5 screening tool and claimed that the questionnaire effectively discriminated between self-assessed severity ratings and patients with confirmed DED. Scores higher than 6 suggest DED, and scores above 12 need a more in-depth investigation.

\section{IMPORTANCE OF LACRITIN}

Lacritin is a glycoprotein produced by the lacrimal gland and is apparent when screening for tear protein secretion factors. It promotes tear production and assists cell proliferation by acting as a mitogen. Lacritin breakdown produces a bactericidal C-terminal fragment, naturally protecting the eye from infections. The deficiency of tears and possible layer "holes" in the ocular surface tear layer stimulates the release of inflammatory cytokines, which consequentially stress epithelial cells in a vicious cycle [2]. Lacritin, in sufficient quantity, restores normality to the tear layer by facilitating epithelial renewal. It does this by targeting surface cells with the help of heparan sulfate proteoglycan syndecan-1. Reduced lacritin levels occur in autoimmune Sjögren's syndrome when ocular cells become stressed. Lacritin restores oxidative phosphorylation and provides respite for surface cells. Karnati et al. [20] discussed the concept of autophagy-damaged or dead cellular tissue being ingested, lysed, and recycled-promoting ocular health.

\section{MANAGEMENT}

There is no cure for DED, with ongoing ophthalmic reviews required for management [2]. Eliciting a full eye history is obligatory. Before an ophthalmological assessment, a small joint review is advisable to exclude major rheumatological diseases. If visual acuity (VA) is correctable to $20 / 20$ with a prior evaluation, any increased refractive error in a new patient with DED needs further investigation. Refraction results beyond a VA of 20/70 in one or both eyes signify a more significant visual problem. Tonometry checks confirming normal intraocular pressures are reassuring. The exception is in patients with a normal-tension glaucoma variant. Schirmer's test, now a century old, measures basal tear secretion volume. Moisture reaching a 10-mm mark on a paper strip within $5 \mathrm{~min}$ is an expected test result.

The tear break-up time (TBUT) is a method for determining an eye's tear film stability and observing evaporative dry eyes. A drop of fluorescein dye is placed on the eye surface. With blinking refrain, the healthy observed rate of TBUT under slit-lamp examination with cobalt blue light should be higher than $10 \mathrm{~s}$; between 5 and $10 \mathrm{~s}$ is marginal, and less than $5 \mathrm{~s}$ is considered low or rapid. Fluorescein can also highlight punctate epithelial erosions on the ocular surface. The National Eye Institute (NEI) Fluorescein Staining Score (FSS) divides the cornea and conjunctiva into five sections. Each 
area's dye uptake is assessed at $0 / 3$ for no staining to $3 / 3$ for complete absorption. The maximum score is $15 / 15$. Amparo et al. [21] introduced a novel computer-assisted automated scoring technique involving imaging, claiming that the corneal fluorescein staining (CFSi) method was more objective and accurate at assessing DED than the FSS system adopted by the NEI.

The slit-lamp biomicroscope provides an operator with a stereoscopic view, magnifying all eye structures in detail, including the DED tear film layer. Several filters employ enhanced views, such as cobalt blue for fluorescein staining and Goldmann tonometry, supplemented by yellow for contrast differentiation. Red-free (green) filters enhance the appearances of blood vessel pathways, hemorrhages, and inflammation. Fleischer rings are also clearly detectable. Heat absorbing, neutral density, and gray light filters all provide patient comfort, with the lattermost used for patient photosensitivity.

Smartphone applications for the eye's anterior segments are increasing in popularity, especially in the emerging age of telemedicine, taking high-resolution photographic images. These are inexpensive, mobile, and more convenient for teaching [22]. Inomata et al. [23] designed a cross-sectional study collating crowdsourced data in Japan using a smartphone app called DryEyeRhythm, enabling the completion of a DED questionnaire. With 21,394 database records, multivariate-adjusted regression analysis identified risk factors for symptomatic and undiagnosed symptomatic dry eye. The study concluded that smartphone data collection could play a role in early prevention or more effective DED interventions.

Foulks and Pflugfelder [24] described new options for noninvasively diagnosing DED, evaluating the tear layer stability and meniscal height, and searching for compositional changes. Lacrimal proteins increase in patients with dry eye and Meibomian gland disorders, thus acting as markers; these include S100AB and A9 peptides [25].

Point-of-care tests are available for eye care offices. The tear osmometer (TearLab) measures osmolarity. Another test, MMP9, InflammaDry (Quidel), measures tear matrix metalloproteinase-9 (MMP-9), which has a high positive predictive value for confirming DED when elevated. When normal osmolarity occurs in a symptomatic patient, it indicates that another subtype of OS disorder is present [26].

\section{TREATMENT}

\section{Strategies}

TFOS DEWS 11 [2] recommends a staged management strategy with four levels:

1. Educational counseling, reassurance, and over-the-counter medications

2. Office-based treatments, specific prescription medications, with additional overnight treatments

3. Oral secretagogues, accompanied by autologous serum eye drops

4. Corticosteroid therapy over some time, with possible surgical intervention

\section{First Steps: Guidance}

Primary health care practitioners (PHCP) are well placed to recommend the first steps in providing DED guidance to reduce or avoid mild risk factors such as prolonged reading, excessive computer screen engagement, prolonged contact lens use, brisk air-conditioning flow, reduced humidity as on commercial flights, and direct exposure to chemicals as in exhaled tobacco smoke which are all temporary [1]. Extended digital device and computer screen use significantly reduce the blink rate and induce eye strain. Certain occupational activities are hazardous, requiring employees to take precautions with care on reduced tear production or increased evaporation rates [27]. Global pollution poses climatic threats of DED occurring on the basis of geographical location. Environmental stresses include prolonged eye exposure in regions with high wind velocity, such as in polar (desiccating) or dusty (desert sand with foreign body risk) areas. Van Setten et al. [28] confirmed a higher prevalence of seasonal- and weather-related DED signs and 
symptoms in a European study. Advancing technology has paradoxically enhanced awareness of visual ergonomics. Anshel [29] coined the term "computer vision syndrome (CVS)" when discussing computer-screen generated visual stress. The adage of taking a 20-s break, every $20 \mathrm{~min}$, and looking at an object 20 feet away is promoted by the American Optometric Association [30]. Adding active blinking 20 times lubricates the ocular surface. The repeated maneuver stimulates the production of a metastable tear film between blinks, enhances clear vision, and maintains ocular service cells' health and turnover [31].

Some medications reduce tear production, mainly some prescribed for hypertension, depression, or antihistamines. PHCPs need to advise patients on side effects when specific prescriptions for the aforementioned conditions are issued. Humidification is essential, together with purposeful, active blinking. Wearing special glasses during work or sunglasses when conditions are windy or warm with bright sunlight effectively protects eyes from OS desiccation. Ophthalmic gels used overnight maintain moisture levels, as are twice-daily applications of artificial tear replacement. Warm eye compresses improve Meibomian gland secretion and sooth surface areas, with additional lid hygiene advised. Bae et al. [32] claimed that high parenteral doses of vitamin $\mathrm{D}$ therapy improved DED by reducing ocular surface inflammation, promoting tear secretion, and reducing tear instability, but this is rarely recommended as initial treatment today.

It is essential to complete a physical assessment for any underlying autoimmune conditions mentioned earlier when considering DED as a diagnosis, to select the appropriate treatment.

\section{Blepharitis Treatment}

Blepharitis is apparent with infected eyelash bases and subsequent inflammation of the lid margins. It is the most common pathological eyelid disorder and is difficult to eliminate once chronic, with a developed biofilm (DEBS) (see
Figs. 3 and 4). Eyelid hygiene is still a fundamentally useful activity. Topical or oral antibiotics, ophthalmic steroid treatment, and calcineurin inhibitors have been used with a modicum of success [33]. Ribeiro et al. [34] completed a systematic review on the effectiveness of using preservative-free artificial tears versus preserved lubricants to treat DED and found no statistically significant difference. A more recent innovation involves autologous serum eye drops (AS) as an option in severe DED when there is no response to other recognized medications. The biochemical components in AS more closely mimic natural tears and act as a lacrimal substitute for OS lubrication [35]. While used as second-line therapy, AS therapy is inconsistent for beneficial outcomes, and further research is needed to establish its value.

\section{Specialist Medications}

Lifitegrast, an anti-inflammatory medication, is US Food and Drug Administration (FDA)-approved for the signs and symptoms of DED. Used for all forms of the eye disorder by specialist ophthalmologists, conditions include keratoconjunctivitis sicca, together with MGD, and consequential inflammatory eye reactions, it inhibits a T lymphocyte function-associated antigen (LFA-1) known as integrin from binding to an intercellular adhesion molecule, ICAM-1 $[36,37]$. Lifitegrast provides rapid onset symptom relief of itching, general eye discomfort, and a foreign body sensation. Side effects may manifest as transient blurring of vision, a stinging sensation, and dysgeusia.

Cyclosporine A (CsA) was discovered in the 1970 s and initially prescribed for solid organ transplant surgery as an immunosuppressant. Experimental ophthalmological trials in the late 1970s [38] progressed to successful human use in preventing corneal allograft reactions by the 1980s, proving useful for settling inflammatory ocular surface disorders [39]. CsA binds to another protein, cyclophilin A, to form a complex molecule modulating immune responses by inhibiting T lymphocyte activity. In 2003, a $0.5 \%$ emulsion of CsA successfully managed DED [40] and continues to be beneficial for 
ocular surface disease [41, 42]. Thermal pulsation therapy for MGD almost doubles patients' TBUT [43]. The mean increase was estimated from 4.5 to $8.5 \mathrm{~s}(P<0.001)$, OSDI score (mean decrease from 50.5 to $41.6[P=0.024])$, and MMP-9 (50\% positive rate pre treatment compared to $26 \%$ positive rate post treatment $[P<0.0001])$. CsA and lifitegrast therapy are used exclusively by specialist ophthalmologists and are not usually initiated by primary health care physicians.

\section{NUTRITIONAL IMPACT}

Eye health benefits, such as reduced irritation with OSD, maintained and preserved vision, and reduced age-related macular degeneration (AMD), can be achieved by a selected nutritional intake. Omega-3 fatty acid-rich foods, such as salmon, mackerel, sardines, and other ocean fishes, while beneficial for general health, were previously thought to improve DED. There is equivocal evidence for vitamins preventing cataract development [44]. Asbell et al. [45] first drew attention to omega-3 being a questionable beneficial supplement in her DREAM study. When joined later by Hussain et al. [46] in a randomized controlled trial, continuing or discontinuing omega-3 fatty acids supplements in patients did not cause significantly worse outcomes for those with DED. Non-seafood consumers can compensate by taking fish oil supplements, vegetarian supplements containing flaxseed or blackcurrant seed oils, or nuts, such as almonds, walnuts, and pistachios. In the AREDS2 trial, Chew et al. [47] determined that leafy greens containing the powerful antioxidants zeaxanthin and lutein also assist with eye health by reducing the chances of AMD. Carotenoid-containing foods in colored fruits and vegetables can benefit eye health, particularly those that also containing vitamin $\mathrm{C}$, vitamin $\mathrm{E}$, zinc, copper, eicosapentanoic acid (EPA), and docosahexanoic acid (DHA). All are proven to reduce the rate of advanced AMD.

Eggs are both lutein-rich and contain high levels of vitamin A. Egg yolks contain approximately 200-250 $\mu \mathrm{g}$ of lutein and are up to three times more bioavailable than comparable vegetable sources. Lean beef eaten in moderation, rich in vitamin A, can boost corneal and conjunctival eye health. Other healthy vitamins include $\mathrm{B}_{6}, \mathrm{~B}_{9}, \mathrm{~B}_{12}$, riboflavin, and niacin, many of which enable consumption of a substantial part of a recommended daily allowance (RDA) of trace elements in commercial cereal products.

\section{CONCLUSION}

DED is a challenging disorder to diagnose and is hard to treat on a first presentation. It continues to be a significant issue in primary care and ophthalmological services, with approximately one-half of those diagnosed not receiving adequate treatment beyond artificial tears. The TFOS DEWS II team [1] report is a significant advance to defining DED, and establishing updated diagnostic parameters. Earlier recognition by primary care family physicians and newer treatments can provide swifter intervention and relief for patients before they advance to more severe symptoms needing referral to an ophthalmologist.

\section{ACKNOWLEDGEMENTS}

The authors express thanks to both Ms. Sinéad O'Rourke, Content Development Specialist, Distributed eLibrary, and Ms. Cathleen de Groot, Manager, User Services, Distributed eLibrary, Weill Cornell Medicine-Qatar, Education City, Qatar Foundation, Doha, Qatar, for their support and assistance.

Funding. This publication was partially funded by Weill Cornell Medicine - Qatar. The Rapid Service Fee was funded by the authors

Authorship. All named authors meet the International Committee of Medical Journal Editors (ICMJE) criteria for authorship for this article, take responsibility for the integrity of the work as a whole, and have given their approval for this version to be published. 
Disclosures. Mohamud A. Verjee has nothing to disclose. Ashley R. Brissette is a consultant to Alcon, Allergan, Inc., Carl Zeiss Medica, and Eyevance Pharmaceuticals, with interests in Alcon and Novartis. Christopher E. Starr is a consultant to and has interests in Allergan, Inc., Essiri LLC, BlephEx, Bruder Healthcare Co., Dompé, Eyevance Pharmaceuticals, Johnson \& Johnson Vision Care, Inc., Kala Pharmaceuticals, Novartis Corp., Quidel Corporation, Sight Sciences, Inc., Sun Pharmaceutical Industries Ltd., Tearlab Corp., Verily, and Sparca.

Compliance with Ethics Guidelines. This article is based on previously conducted studies and does not contain any studies with human participants or animals performed by any of the authors.

Data Availability. Data sharing does not apply to this article as no datasets were generated or analyzed during the current review.

Open Access. This article is licensed under a Creative Commons Attribution-NonCommercial 4.0 International License, which permits any non-commercial use, sharing, adaptation, distribution and reproduction in any medium or format, as long as you give appropriate credit to the original author(s) and the source, provide a link to the Creative Commons licence, and indicate if changes were made. The images or other third party material in this article are included in the article's Creative Commons licence, unless indicated otherwise in a credit line to the material. If material is not included in the article's Creative Commons licence and your intended use is not permitted by statutory regulation or exceeds the permitted use, you will need to obtain permission directly from the copyright holder. To view a copy of this licence, visit http://creativecommons.org/licenses/by$\mathrm{nc} / 4.0 /$.

\section{REFERENCES}

1. Craig JP, Nichols KK, Akpek EK, et al. TFOS DEWS II definition and classification report. Ocul Surf.
2017;15(3):276-83. https://doi.org/10.1016/j.jtos. 2017.05.008.

2. Dry Eye Workshop (DEWS). The definition and classification of dry eye disease: report of the Definition and Classification Subcommittee of the International Dry Eye Workshop. Ocul Surf. 2007;5(2):75-92. https://doi.org/10.1016/s15420124(12)70081-2.

3. Behrens A, Doyle JJ, Stern L, et al. Dysfunctional tear syndrome: a Delphi approach to treatment recommendations. Cornea. 2006;25(8):900-7. https://doi.org/10.1097/01.ico.0000214802.40313. fa.

4. Rynerson JM, Perry HD. DEBS-a unification theory for dry eye and blepharitis. Clin Ophthalmol. 2016;10:2455-67. https://doi.org/10.2147/OPTH. S114674.

5. Starr CE. Anterior blepharitis showing scurf. Weill Cornell Medicine, New York; 2020.

6. Starr CE. Anterior blepharitis showing keratinization. Weill Cornell Medicine, New York; 2020.

7. Starr CE. Meibography_showing Meibomian gland dysfunction (MGD). Weill Cornell Medicine, New York; 2020.

8. Schaumberg DA, Sullivan DA, Buring JE, Dana MR. Prevalence of dry eye syndrome among US women. Am J Ophthalmol. 2003;136(2):318-26. https://doi. org/10.1016/s0002-9394(03)00218-6.

9. Rouen PA, White ML. Dry eye disease: prevalence, assessment, and management. Home Healthc Now. 2018;36(2):74-83. https://doi.org/10.1097/NHH. 0000000000000652 .

10. Gupta PK, Drinkwater OJ, VanDusen KW, Brissette AR, Starr CE. Prevalence of ocular surface dysfunction in patients presenting for cataract surgery evaluation. J Cataract Refract Surg. 2018;44(9): 1090-6. https://doi.org/10.1016/j/jcrs.2018.06.026.

11. Starr CE, Gupta PK, Farid M, et al. An algorithm for the preoperative diagnosis and treatment of ocular surface disorders. J Cataract Refract Surg. 2019;45(5):669-84. https://doi.org/10.1016/j.jcrs. 2019.03.023.

12. Ekker MS, Janssen S, Seppi K, et al. Ocular and visual disorders in Parkinson's disease: common but frequently overlooked. Parkinsonism Relat Disord. 2017;40:1-10. https://doi.org/10.1016/j.parkreldis. 2017.02.014.

13. Wolffsohn JS, Craig JP, Vidal-Rohr M, Huarte ST, Ah Kit L, Wang M. Blink test enhances ability to screen for dry eye disease. Cont Lens Anterior Eye. 
2018;41(5):421-5. https://doi.org/10.1016/j.clae. 2018.06.003.

14. American Academy of Ophthalmology. Imagefluorescein stain highlighting ocular surface damage. 2020. http://www.aao.org. Accessed 14 March 20, 2020 .

15. Starr CE. Dry eye disease-fluorescein staining showing localized ocular surface damage. Weill Cornell Medicine, New York; 2020.

16. Na KS, Han K, Park YG, Na C, Joo CK. Depression, stress, quality of life, and dry eye disease in Korean women: a population-based study. Cornea. 2015;34(7):733-8. https://doi.org/10.1097/ICO. 0000000000000464 .

17. Schiffman RM, Walt JG, Jacobsen G, Doyle JJ, Lebovics G, Sumner W. Utility assessment among patients with dry eye disease. Ophthalmology. 2003;110:1412-9. https://doi.org/10.1016/S01616420(03)00462-7.

18. Amparo F, Schaumberg DA, Dana R. Comparison of two questionnaires for dry eye symptom assessment: The Ocular Surface Disease Index and the Symptom Assessment in Dry Eye. Ophthalmology. 2015;122(7):1498-503. https://doi.org/10.1016/j. ophtha.2015.02.037.

19. Chalmers RL, Begley CG, Caffery B. Validation of the 5-Item Dry Eye Questionnaire (DEQ-5): discrimination across self-assessed severity and aqueous tear deficient dry eye diagnoses. Cont Lens Anterior Eye. 2010;33(2):55-60. https://doi.org/10. 1016/j.clae.2009.12.010.

20. Karnati R, Talla V, Peterson K, Laurie GW. Lacritin and other autophagy associated proteins in ocular surface health. Exp Eye Res. 2016;144:4-13. https:// doi.org/10.1016/j.exer.2015.08.015.

21. Amparo F, Wang H, Yin J, Marmalidou A, Dana R. Evaluating corneal fluorescein staining using a novel automated method. Invest Ophthalmol Vis Sci. 2017;58(6):BIO168-73. https://doi.org/10. 1167/iovs.17-21831.

22. Chan JB, Ho HC, Ngah NF, Hussein E. DIYsmartphone slit-lamp adaptor. J Mob Technol Med. 2014;3(1):16-22. https://doi.org/10.7309/jmtm.3.1. 4.

23. Inomata $\mathrm{T}$, Iwagami $\mathrm{M}$, Nakamura $\mathrm{M}$, et al. Characteristics and risk factors associated with diagnosed and undiagnosed symptomatic dry eye using a smartphone application. JAMA Ophthalmol. 2019;138(1):58-68. https://doi.org/10.1001/ jamaophthalmol.2019.4815.
24. Foulks GN, Pflugfelder SC. New testing options for diagnosing and grading dry eye disease. Am J Ophthalmol. 2014;157(6):1122-9. https://doi.org/ 10.1016/j.ajo.2014.03.002.

25. Tong L, Zhou L, Beuerman RW, Zhao SZ, Li XR. Association of tear proteins with Meibomian gland disease and dry eye symptoms. Br J Ophthalmol. 2011;95(6):848-52. https://doi.org/10.1136/bjo. 2010.185256 .

26. Brissette AR, Drinkwater OJ, Bohm KJ, Starr CE. The utility of a normal tear osmolarity test in patients presenting with dry eye disease like symptoms: a prospective analysis. Cont Lens Anterior Eye. 2019;42(2):185-9. https://doi.org/10.1016/j.clae. 2018.09.002.

27. Lee JH, Lee W, Yoon JH, Seok H, Roh J, Won JU. Relationship between symptoms of dry eye syndrome and occupational characteristics: the Korean National Health and Nutrition Examination Survey 2010-2012. BMC Ophthalmol. 2015;15:147. https://doi.org/10.1186/s12886-015-0147-3.

28. van Setten G, Labetoulle M, Baudouin C, Rolando M. Evidence of seasonality and effects of psychrometry in dry eye disease. Acta Ophthalmol. 2016;94(5):499-506. https://doi.org/10.1111/aos. 12985.

29. Anshel JR. Visual ergonomics in the workplace. AAOHN J. 2007;55(10):414-22. https://doi.org/10. $1177 / 216507990705501004$.

30. American Optometric Association. https://www. aoa.org/AOA/Images/Patients/Eye\%20Conditions/ 20-20-20-rule.pdf. Accessed 14 August 2020.

31. Lemp MA. Advances in understanding and managing dry eye disease. Am J Ophthalmol. 2008;146(3):350-6. https://doi.org/10.1016/j.ajo. 2008.05.016.

32. Bae SH, Shin YJ, Kim HK, Hyon JY, Wee WR, Park SG. Vitamin D supplementation for patients with dry eye syndrome refractory to conventional treatment. Sci Rep. 2016;6:33083. https://doi.org/10. 1038/srep33083.

33. Duncan K, Jeng BH. Medical management of blepharitis. Curr Opin Ophthalmol. 2015;26(4): 289-94. https://doi.org/10.1097/ICU. 0000000000000164 .

34. Ribeiro MVMR, Barbosa FT, Ribeiro LEF, Sousa-Rodrigues $\mathrm{CF}$, Ribeiro EAN. Effectiveness of using preservative-free artificial tears versus preserved lubricants for the treatment of dry eyes: a systematic review. Arq Bras Oftalmol. 2019;82(5):436-45. https://doi.org/10.5935/0004-2749.20190097. 
35. Pan Q, Angelina A, Marrone M, Stark WJ, Akpek EK. Autologous serum eye drops for dry eye. Cochrane Database Syst Rev. 2017;2(2):CD009327. https:// doi.org/10.1002/14651858.cd009327.pub3.

36. Semba CP, Gadek TR. Development of lifitegrast: a novel T-cell inhibitor for the treatment of dry eye disease. Clin Ophthalmol. 2016;10:1083-94. https://doi.org/10.2147/OPTH.S110557.

37. Tauber J, Karpecki P, Latkany R, et al. Lifitegrast ophthalmic solution $5.0 \%$ versus placebo for treatment of dry eye disease: results of the randomized phase III OPUS-2 study. Ophthalmology. 2015;122(12):2423-31. https://doi.org/10.1016/j. ophtha.2015.08.001.

38. Coster DJ, Shepherd WF, Fook TC, Rice NS, Jones BR. Prolonged survival of corneal allografts in rabbits treated with cyclosporin A. Lancet. 1979;2(8144):688-9. https://doi.org/10.1016/ s0140-6736(79)92082-8.

39. Holland EJ, Olsen TW, Ketcham JM, et al. Topical cyclosporin A in the treatment of anterior segment inflammatory disease. Cornea. 1993;12(5):413-9. https://doi.org/10.1097/00003226-19930900000008 .

40. Tatlipinar S, Akpek EK. Topical ciclosporin in the treatment of ocular surface disorders. Br J Ophthalmol. 2005;89(10):1363-7. https://doi.org/10. 1136/bjo.2005.070888.

41. Boboridis KG, Konstas AGP. Evaluating the novel application of cyclosporine $0.1 \%$ in ocular surface disease. Expert Opin Pharmacother. 2018;19(9): 1027-39. https://doi.org/10.1080/14656566.2018. 1479742 .
42. Boboridis KG, Kozeis N, Konstas AG. Revisiting ocular allergy: evaluating symptoms, benzalkonium chloride and efficacy of topical ketotifen 0.025 . Ocul Immunol Inflamm. 2020;28(2):188-90. https://doi.org/10.1080/09273948.2018.1560478.

43. Kim MJ, Stinnett SS, Gupta PK. Effect of thermal pulsation treatment on tear film parameters in dry eye disease patients. Clin Ophthalmol. 2017;11: 883-6. https://doi.org/10.2147/OPTH.S136203.

44. McCusker MM, Durrani K, Payette MJ, Suchecki J. An eye on nutrition: the role of vitamins, essential fatty acids, and antioxidants in age-related macular degeneration, dry eye syndrome, and cataract. Clin Dermatol. 2016;34(2):276-85. https://doi.org/10. 1016/j.clindermatol.2015.11.009.

45. Asbell PA, Maguire MG, DREAM Study Research Group. Why DREAM should make you think twice about recommending omega-3 supplements. Ocul Surf. 2019;17(4):617-8. https://doi.org/10.1016/j. jtos.2019.08.003.

46. Hussain M, Shtein RM, Pistilli M, et al. The Dry Eye Assessment and Management (DREAM) extension study-a randomized clinical trial of withdrawal of supplementation with omega-3 fatty acid in patients with dry eye disease. Ocul Surf. 2020;18(1): 47-55. https://doi.org/10.1016/j.jtos.2019.08.002.

47. Age-Related Eye Disease Study 2 (AREDS2) Research Group, Chew EY, Clemons TE, et al. Secondary analyses of the effects of lutein/zeaxanthin on agerelated macular degeneration progression: AREDS2 report No. 3. JAMA Ophthalmol. 2014;132(2): 142-9. https://doi.org/10.1001/jamaophthalmol. 2013.7376. 